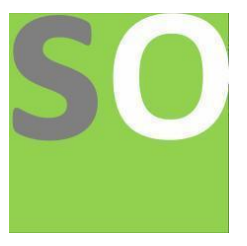

Article title: Digitification: Why the World Community Needs a New Term

Authors: Kseniia Nazarova[1]

Affiliations: Orel State University[1]

Orcid ids: 0000-0003-2477-2673[1]

Contact e-mail: kn@dutiap.com

License information: This work has been published open access under Creative Commons Attribution License http://creativecommons.org/licenses/by/4.0/, which permits unrestricted use, distribution, and reproduction in any medium, provided the original work is properly cited. Conditions, terms of use and publishing policy can be found at https://www.scienceopen.com/.

Preprint statement: This article is a preprint and has not been peer-reviewed, under consideration and submitted to ScienceOpen Preprints for open peer review.

DOI: 10.14293/S2199-1006.1.SOR-.PPERJWB.v1

Preprint first posted online: 29 September 2021

Keywords: Digitification, sexual objectification, rating system, objectification, rating score, digitalization 


\title{
DIGITIFICATION
}

\section{Digitification: Why the World Community Needs a New Term}

\section{Kseniia Nazarova}

\begin{abstract}
For many people, the process of assessing one person to another is negative and can be defined as objectification, due to subjective perception and social attitudes. But in society, there is still a need to assess the attributes of the subject and therefore, in order to separate a bad assessment from a good one and not cause a public outcry, it is necessary to introduce a new term digitification, which carries a neutral connotation and does not violate people's personal boundaries. Such a term will make it possible to separate the concepts of objectification and digitification, not to confuse them and not to ascribe to each other inappropriate characteristics. Digitification is the process of assessing the attributes of a certain person on a voluntary basis by another person or artificial intelligence, based on objective criteria created for a particular rating system. Moreover, rating systems are different and have different functions, and therefore the rating system must be correctly selected in order to be more accurate and effective. Various online services and trading platforms have their own rating systems, their business is successful and relationships with customers are well established. Moreover, there is a whole state in the world in which the rating system is an integral part of society and it also shows good results. This article was created with the aim of introducing a new term digitification into everyday life that can change the course of human thinking for the better and open up new possibilities in the field of measurement systems through ratings and reviews.
\end{abstract}




\section{Keywords}

Digitification, objectification, sexual objectification, rating system, rating score, digitalization

\section{Introduction}

In this article, the key goal is to show and make it clear that the world does not stand still. It develops and does it successfully with high speed using new technologies. The modern world and information technologies are already inseparable from each other and step by step digitalization becomes something commonplace for a person.

But along with information technologies, which are becoming a part of people's lives, various social problems appear, which people themselves can solve only. One of these problems will be presented in this article. This problem is deep and difficult to eradicate. This problem is associated with gender inequality, discrimination against women and their objectification (including sexual objectification). But at the same time, this problem is associated with information technology and the process of digitalization.

With the advent of new technologies, a large number of platforms and services have appeared that offer to rate their goods and services. People also can be assessed, but is any rating necessary and useful? This is a topic of debate among those who consider the assessment for a person to be an objectification process and those who need to get a certain rating in order to assess their attributes and improve further.

This is not about the person in general, this is about his qualities, skills and abilities. What is the manifestation of objectification in assessing the skills of a taxi driver? Why can't people rate the quality of the work of a nail artist in the 
beauty sphere? And what is wrong if people independently want to rate not only their skills, but also external qualities, which, for example, are necessary in the beauty sphere or the sphere of modeling. The answers for these and many other questions can be found in this article.

\section{Objectification, self-objectification and sexual objectification}

Learning a new term, it is necessary to understand what objectification is, because objectification is the opposite of the new term digitification. If objectification is defined correctly, then it is easy to understand the meaning of digitification. There are different definitions of the word objectification from different points of view, but in the classical understanding of social philosophy objectification is the act of treating a person, or sometimes an animal, as an object or a thing. It is a part of dehumanization, the act of disavowing the humanity of others. ${ }^{1}$

According to Martha Nussbaum - an American philosopher and the current Ernst Freund Distinguished Service Professor of Law and Ethics at the University of Chicago - a person is objectified if one or more of the following properties are applied to them: ${ }^{2}$

1. Instrumentality - treating the person as a tool for another's purposes

2. Denial of autonomy - treating the person as lacking in autonomy or self-determination

3. Inertness - treating the person as lacking in agency or activity

4. Fungibility - treating the person as interchangeable with (other) objects

5. Violability - treating the person as lacking in boundary integrity and violable, "as something that it is permissible to break up, smash, break into."

\footnotetext{
${ }_{1}^{1}$ Arluke, Arnold (1988). "Sacrificial Symbolism in Animal Experimentation: Object or Pet?". Anthrozoös: A Multidisciplinary Journal of the Interactions of People and Animals. 2 (2): 98-117.

${ }^{2}$ Nussbaum, Martha C. (1985). "Objectification". Philosophy \& Public Affairs. 24 (4): 249-291.
} 
6. Ownership - treating the person as though they can be owned, bought, or sold

7. Denial of subjectivity - treating the person as though there is no need for concern for their experiences or feelings ${ }^{3}$

Rae Helen Langton - an Australian-British professor of philosophy - proposed 3 additional properties to be added to Nussbaum's list:

1. Reduction to body - the treatment of a person as identified with their body, or body parts

2. Reduction to appearance - the treatment of a person primarily in terms of how they look, or how they appear to the senses

3. Silencing - the treatment of a person as if they are silent, lacking the capacity to speak ${ }^{4}$

Although the word objectification itself is several centuries old, and the phenomenon is even more, the modern social agenda has enriched the dictionary with this concept with another, different from the classical meaning, which is accepted negatively. This is a term of sexual objectification or simply SO.

Both men and women can be sexually objectified, but the concept of SO is mainly associated with women, and it is an important idea in many feminist theories and psychological theories derived from them. Sexual objectification of women contributes to gender inequality, and many psychologists equate objectification with a range of physical and mental health risks in women.

The sexual objectification of women involves them being viewed primarily as an object of male sexual desire, rather than as a whole person. ${ }^{5}$ Although opinions differ as to which situations are objectionable, many people see the objectification

\footnotetext{
${ }^{3}$ Nussbaum, Martha C. (1985). "Objectification". Philosophy \& Public Affairs. 24 (4): 257.

${ }^{4}$ Langton, Rae Helen (2009). Sexual Solipsism: Philosophical Essays on Pornography and Objectification (1st ed.). Oxford: Oxford University Press. pp. 228-229.

${ }^{5}$ Barry, Kathleen (1984). "Pornography: the ideoloy of cultural sadism". In Barry, Kathleen (ed.). Female sexual slavery. New York London: NYU Press. p. 247.
} 
of women in the depictions of women in advertising, art, media and pornography, and women being brazenly evaluated or judged sexually or aesthetically in public spaces and events.

Some feminists and specialists argue that SO can lead to negative psychological effects such as eating disorders, depression and sexual dysfunction. Moreover, they can cause a negative perception of themselves due to the fact that their intellectual abilities and competence are not currently recognized and will never be recognized by society. It was also found that sexual objectification of women and girls negatively affects their productivity, academic performance, self-esteem and the level of position in school, university or workplace.

Pro-feminist cultural critics such as Robert Jensen and Sut Jhally accuse mass media and advertising of promoting the objectification of women to help promote goods and services, ${ }^{6}$ and both TV and movie industries are commonly accused of normalizing the sexual objectification of women.

The first mentions of the objectification of women appeared quite a long time ago. For example, in the French Enlightenment there was a debate whether a woman's breasts were merely a sensual enticement or rather a natural gift. In Alexandre Guillaume Mouslier de Moissy's 1771 play The True Mother (La Vraie Mère), the main character reproaches her husband for treating her as an object for his sexual gratification: "Are your senses so gross as to look on these breasts - the respectable treasures of nature - as merely an embellishment, destined to ornament the chest of women?"7

Issues of SO first became problematic in the 1970s by feminist groups. Since then, it has been argued that the phenomenon of female SO has increased dramatically

\footnotetext{
${ }^{6}$ Jhally, Sut (director) (1997). Dreamworlds II: desire, sex, power in music (Documentary). USA: Media Education Foundation.

${ }^{7}$ Schama, Simon (1989). "The cultural construction of a citizen: II Casting roles: children of nature". In Schama, Simon (ed.). Citizens: a chronicle of the French Revolution. New York: Knopf Distributed by Random House.
} 
since its problematization in all levels of life, and has resulted in negative consequences for women, especially in the political sphere. However, a rising form of new third-wave feminist groups have also taken the increased objectification of women as an opportunity to use the female body as a mode of power. ${ }^{8}$

Male SO also implies that men are seen primarily as an object of sexual desire, but not as a person in general. Psychologist Harold Lyon suggests that men's liberation is a necessary step toward women's liberation. ${ }^{9}$

Instances where men may be viewed as sex objects by women include advertisements, music videos, films, television shows, beefcake calendars, women's magazines, male strip shows, and clothed female/nude male (CFNM) events. ${ }^{10}$ Women purchase and consume pornography as well as men.

Within gay male communities, men are often objectified by other men. ${ }^{11}$ Discussing negative effects of objectification is met with considerable resistance in the community. The sexual objectification of men of color may force them to play specific roles in sexual encounters that are not necessarily of their own choosing. ${ }^{12}$ Research suggests that the psychological effects of objectification on men are similar to those of women, leading to negative body image among men. ${ }^{13}$

Male bodies as well as female bodies have become objectified, and even more than they were before. But it is worth noting the fact that this objectification is not so widespread due to the well-established view of society on the objectification of women.

\footnotetext{
${ }^{8}$ Heldman, Caroline (August 2011). "Sexualizing Sarah Palin: the social and political context of the sexual objectification of female candidates". Sex Roles. 65 (3): 156-164.

${ }^{9}$ Lyon, Harold C. (1977). Tenderness Is Strength (first ed.). New York: Harper \& Row.

10 "Sports, gym classes, team initiations and events". Sensations4women.com. 26 January 1998.

Retrieved 1 August 2012.

11 Teunis, Niels (May 2007). "Sexual objectification and the construction of whiteness in the gay male community". Culture, Health \& Sexuality. 9 (3): 263-275.

12 Teunis, Niels (2007). "Sexual objectification and the construction of whiteness in the gay male community". Culture, Health \& Sexuality. 9 (3): 263-275.

${ }^{13}$ Neimark, Jill (1 November 1994). "The beefcaking of America". Psychology Today. Sussex Publishers.

Retrieved 1 August 2012.
} 
Male sexual objectification has been found in $37 \%$ of advertisements featuring men's body parts to showcase a product. ${ }^{14}$ Male and female sexual objectification have common similarities. For example, male objectification also leads men to body shame, eating disorders, and the pursuit of excellence.

You can often see that male actors who appear in various TV shows or movies and series are in excellent physical shape and have "perfect" bodies. As a rule, such men win the main roles, and therefore the hearts of the fans. When society is subjected to men who do not have ideal bodies, we typically see them as the comic relief. A man out of shape in the lead role is rare. Leanne Dawson writes that "There are temporal, cultural and geographical 'norms' of gender and other aspects of identity, which are often incorrectly considered to be inherent or natural."15 In the media, sexual objectification has been used as a way to sell products to the general public. ${ }^{16} \mathrm{SO}$ has been used as a marketing strategy for several decades, according to the Journal of Advertising. This strategy is used to sell products that make men look and feel attractive and desirable to women. It is argued that such a strategy helps to sell successfully while gaining public attention. The magazine also states that explicit ads are more effective in marketing than implicit ads. Summarizing all of the above, the following conclusions can be drawn:

1. Objectification is a term that carries a negative character and, of course, has a negative impact on entire groups of society

2. Objectification refers to different groups of society and is based on gender inequality

\footnotetext{
${ }^{14}$ Davidson, M. Meghan; Gervais, Sarah J.; Canivez, Gary L.; Cole, Brian P. (April 2013). "A psychometric examination of the Interpersonal Sexual Objectification Scale among college men". Journal of Counseling Psychology. 60 (2): 239-250.

${ }^{15}$ Dawson, Leanne (2015). "Passing and policing: controlling compassion, bodies and boundaries in Boys Don't Cry and Unveiled/Fremde Haut". Studies in European Cinema. 12 (3): 205-228.

${ }^{16}$ Zimmerman, Amanda; Dahlberg, John (1 March 2008). "The Sexual Objectification of Women in Advertising: A Contemporary Cultural Perspective". Journal of Advertising Research. 48 (1): 71-79.
} 
3. In most cases, sexual objectification is directed at the female sex, but male sexual objectification also takes place

4. SO causes problems with both physical and psychological health, as well as complexes, low self-esteem, feelings of inferiority and fears of oneself, the opposite sex or society as a whole

5. The process of objectification is violent and cannot be controlled. Such a process takes place without any permission, because it is a subjective opinion of a person, and therefore it is very difficult to eradicate it.

\section{Why the world community needs a new term digitification and what distinguishes it from the terms existed before}

The modern world, as everyone knows, is full of surprises and changes. Time in this world is running out at an incredible speed, and at the same time society and its views are changing as well. And if a couple of centuries ago, modern global problems did not even exist or did not seem so global, now they are widely publicized and even cause public discord. This is about the problems of gender inequality, discrimination against certain groups of society and the problems of their perception by other groups. Objectification is one of such problems as described above.

The main task of this paper is to prove that, in parallel with the negative aspects of objectification, there are also positive ones, which, unfortunately, are lost among erroneous opinions and misconceptions about the concepts of beauty and its assessment. Exactly for these purposes a new term that contains a neutral connotation and does not defile any social groups and their views should appear in the modern world, along with the terms objectification, gender inequality, sexism, beautification and digitalization. 
The new term is called digitification. This word does not exist as a term and is absent in dictionaries, and therefore can be used to form a new concept.

Digitification should be characterized as an assessment of the qualities of a subject based on objective criteria. As a rule these criteria are already embedded in the rating system itself and can be specific questions or a clearly formulated scale for assessing attributes.

Unlike objectification, in the process of digitification the subject himself is not objectified. Instead of that, his qualities, properties and roles are objectified. For example, the driving skills of a taxi driver, or the quality of the work of a consultant in a store. Such qualities can be the ability of a football player to make an assist or the skill of a chef to bring meat to medium rare.

The process of digitification can be used to measure, rate and therefore change these qualities. Or even improve them in order to become better or to provide someone with high quality services.

Also, unlike objectification, digitification is not a derogatory process in relation to the subject, on condition of voluntary consent. Voluntary consent can be expressed both orally and in writing. The terms of use, employment contract and any other document that is voluntarily signed by a person can be considered as a written consent.

Digitification can be done by other subjects, algorithms and artificial intelligence.

\section{Why do people need a rating system?}

Sites like Yelp and TripAdvisor grew out of the rating systems of the last century. These include Michelin guides that first started rating restaurants in the 1920s, film grading that also began in the 1920s and hotel star rating systems that got going in the 1950s. Initially, these and many other ratings were based on an objective list of criteria and were even conducted by experts in their fields, unlike modern ones. 
But both the first ratings and their modern analogs have one similarity - they were all aimed to provide customers with useful product or service information that they needed.

The development of ratings has become a whole industry with the advent of the Internet and social networks, and now almost anyone can become a critic of anything, for example business, beauty services, books, hotels, cafes and restaurants and, of course, taxi drivers.

These rating systems serve two both customers and businesses at the same time. They also give "the crowd" tremendous influence. It starts getting scary while realising that any user has power over a business. For instance 1 low rating is too much for an Uber driver, so he or even his profile can be deactivated immediately. For every star lost on Yelp, the restaurant's revenue drops by $5-10 \%$, and if only one star remains, the restaurant owner can pack up his belongings because the restaurant is likely to be bankrupt.

Ratings are not just an assessment nowadays, this is a certain lifestyle when you choose and buy all kinds of products according to ratings and reviews. It is impossible to imagine the modern world without both online and offline ratings everywhere. And the more successful the business, the higher its score, which means the higher the client's confidence in the business or service.

\section{Rating scale and its characteristics}

For this paper, it is also very important to understand the issue of the rating scale. What it is, what it is used for and what types of it are.

A rating scale is a set of categories designed to elicit information about a quantitative or a qualitative attribute. ${ }^{17}$

\footnotetext{
${ }^{17}$ Paul F.M. Krabbe, in The Measurement of Health and Health Status, 2017
} 
In this case, the attribute is various characteristics of the subject that needs to be assessed. For example, speaking of a plumber or an electrician, the attributes are the speed and quality of his work. For a nail artist, in addition to quality and speed, an attribute may be the ability to draw something on nails well. The examples are rather exaggerated, but quite clear and understandable. A rating scale is a method that requires the rater to assign a value, sometimes numeric, to the rated object, as a measure of some rated attribute.

Rating scales can be different. Here is their classification:

- Numeric Rating Scale

- Verbal Rating Scale

- Visual Analogue Scale

- Likert

- Graphic rating scale

- Descriptive graphic rating scale

Rating scales are usually used online in order to provide indications of consumer opinions of services or products. Everyone has visited websites at least once that use a rating scale to measure something. For example, IMDb, Epinions.com, Yahoo! Movies, Amazon.com, BoardGameGeek and TV.com use a rating scale from 0 to 100 in order to obtain "personalised film recommendations". Most websites allow their users to take part in the voting only once, though there are such exceptions as Ratings.net, which allows users to rate products in relation to several qualities.

There is one more rating system such as a binary rating system. It is simple and many users advocate binary rating over a scale rating because it is less ambiguous. Common social scores such as "like" and "follow" should be considered a form of binary rating (with like vs no like). However, using "like" and "follow" can only be used to rank items, but it is not enough for users to compare items with. 
In general, a binary rating system is suitable for sites that do not require complex review such as Reddit, Stack Overflow to simply separate good quality contents from the content pool. In terms of user experience, binary rating is easy to capture because it has less options and it's unambiguous. It can be a challenge to display the binary ratings in a visually appealing way but it is certainly possible.

Overall, binary rating is more user friendly when it comes to capturing and interpreting the feedback, however average users are not too used to seeing binary ratings especially on an online product marketplace.

5 and 10-point rating system can be found everywhere. It is a good and clear rating scale that's being used by popular marketplace sites apps. Because this system is so common, it is intuitive to average users in terms of how they interpret and cast the ratings. Visually, it is easy for users to scan and compare a list of ratings at once. Lastly, scale rating is extremely powerful and flexible because you can obtain feedback with great detail by increasing the number of scale level.

Thus, it becomes obvious that the more detailed the rating scale, the more detailed the rating and the result as a whole. The binary scoring system is simple, but at the same time primitive. It is very abstract and therefore not accurate. The 10-point rating system is much more accurate and qualitative, so the services that use it receive much more accurate data and a much more objective rating.

\section{Uber and Lyft as an unconscious integration of digitalization in the modern world}

Perhaps the first thing that comes to mind in the context of digitalization is Uber, which has taken over the world and has become the world's largest service for finding, calling and paying for a taxi.

Uber has both a driver and a rider rating system. The Uber driver rating system allows passengers to rate their experiences and give feedback since the launch of 
an application in the United States and Canada. The Uber rider rating system is an extension of that. It gives drivers the ability to rate their own experiences in the driver app and warn or recommend passengers to future drivers.

Uber rider ratings use a 5 point rating scale in the form of stars. Uber driver has the same number of stars as the driver ratings. Obviously 5 is the highest score and 1 is the minimum score. Every time you take a trip in an Uber, you give the driver a score, and in return, they do the same for you.

Usually Uber drivers are offered to consider 3 factors when rating passengers:

1. Short wait times

2. Courtesy

3. Safety

Everything is clear with the rating system and its scale. But there is the main question that is interesting within the framework of the study, but which the average user does not think about, taking the rating system in the mobile application as something commonplace: why does Uber have rider ratings? The point is that Uber manages its people with algorithms, and ratings are a disciplinary method. With the help of the rating system, drivers and passengers can rate each other on a five-point scale after each trip, and it is worth noting how this system works: it works in both directions. Echoing Sauder and Espeland's (2009) Foucauldian analysis of law school rankings, Uber's rating system enables remote platform surveillance by transforming categorical differences among drivers into numerical, user-generated ratings that can be easily circulated. ${ }^{18}$ Each driver is a separate case that can be individually assessed, complained and even punished with the help of the assessment.

\footnotetext{
${ }^{18}$ Chan, Ngai Keung. 2019. The Rating Game: The Discipline of Uber's User-Generated Ratings. Surveillance \& Society 17(1/2): 185.
} 
Ratings evince the idea that drivers' performance during each ride is comparable in accordance with "community guidelines". In its "community guidelines," Uber equates a high rating with "good" and "professional" services. What is more, Uber ratings define certain standards, such as showing friendliness and offering certain amenities to passengers. Those who do not conform to the standard implicit in ratings risk being punished.

In the 2015 Federal Trade Commission workshop on the sharing economy, Uber made explicit its design rationale for the rating system:

This rating system does three critical things:

1. Incentivizes high quality service

2. Establishes accountability

3. Promotes courteous conduct, and helps to mitigate the discrimination that is all too common in traditional for-hire transportation ${ }^{19}$

Uber believes that continuous monitoring is beneficial for both the passenger and the driver, as the assessment allows both parties to behave appropriately and prevent unpleasant incidents. In the event of a breach of conduct, either party may be held liable. In this way, the rating system promotes "a safe and respectful work environment for drivers". ${ }^{20}$

Simply put, Uber has ratings for both riders and drivers as part of the company's mission to be safe and respectful to all. This is their policy and they adhere to it, improving algorithms and criteria so that the assessment is as accurate and objective as possible. The mutual rating system ensures the rider's safety as they can report unsafe drivers, while rider ratings help protect other drivers from disrespectful, dangerous, or unpredictable riders. If your score is too low, you might have to wait longer or even be removed from the Uber platform.

\footnotetext{
${ }^{19}$ Chan, Ngai Keung. 2019. The Rating Game: The Discipline of Uber's User-Generated Ratings. Surveillance \& Society 17(1/2): 185.

${ }_{20}$ Uber's Community Guidelines 2015. p. 5
} 
There is another world's largest transportation company which is called Lyft. Its mission is “To improve people's lives with the world's best transportation." Uber and Lyft work the same way. Lyft as well as Uber offers passengers to rate their drivers from 1 to 5 stars after each trip has been completed. A driver is also allowed to rank their passengers. In addition to the rating, riders are allowed to leave feedback for the drivers.

There are many purposes for using a rating system. Rating scales allow the researcher to measure the opinions and behaviors of respondents in a quantitative manner. The number system is used universally as a method of measurement. It is simple to use and almost all cultures around the world are familiar with the standard number system and have seen a 1-10 rating scale before.

Thus, it is worth paying attention to two very important factors:

- Digitalization in the form of an Uber or Lyft rating system in their mobile applications is a peaceful way to make the life of a consumer and a person providing services better and safer

- The process of exchanging and getting ratings between a consumer and a service provider is voluntary, because when applying for a job or getting an application to the phone, employees sign a contract, and users accept the Terms of Use. A person is assessed only by his certain attributes, namely as a driver with his driving skills or as a passenger with his dignified behavior. The person in this case is not objectified

- The aforementioned services use a non-binary scoring system to make the score as accurate as possible, and therefore objective Therefore, it is a priori incorrect to say that the process of digitalization in the form of ratings is negative. Such ratings are a personal and voluntary choice of everyone. That is why digitification term is necessary, because it accurately 
describes the entire process of evaluating oneself as a client or a user, without affecting the process of objectification.

\section{Digitalization and online shopping platform represented by eBay as an example}

Transport companies are and will be popular with people all over the world, despite the frequency of use. Someone needs a taxi a couple of times a month, when they go to their friends for a glass of wine, and then cannot use their car or do not want to use public transport. Some people take a taxi on weekends to get from the bar to their home safely, quickly and comfortably. And someone uses a taxi every day, moving around the city from one business meeting to another. Transport companies are definitely in demand, but there is something that is in demand even much more and among almost the entire population of our planet. These are stores.

We all buy groceries and household goods, a little less often we buy clothes, shoes and accessories, and even less often we do large household purchases. And in the modern world, when almost every person has the Internet, we order all of the above products online without leaving our home, while automatically and almost unconsciously assessing the quality of service from the good itself to delivery. One of such online shopping platforms is eBay.

An eBay star rating and feedback score indicate both a seller's experience and their level of customer service. It represents how high your sales are and how much positive feedback you have received. ${ }^{21}$

Every time a seller earns feedback, they can gain or lose points:

1. The user earns 1 point for each positive rating

2. The user loses 1 point for every negative rating

${ }^{21}$ Chris Dunne. eBay Stars: What They Mean and How to Get Them. Blog.edesk.com. 
3. A neutral rating isn't worth any points

The feedback score usually shows how many points a seller has received, based on this system. Positive ratings - negative ratings $=$ Feedback score.

For a long time, eBay's success has been equated with consumer confidence in the platform. It included payment security, successful dispute resolution and a rating system. The eBay rating system, in which buyers and sellers rate each other, was immediately added to the service as soon as it started working. This measure was introduced so that there were as few cases of fraud on the platform as possible. The rating system is still in effect, because it has become an integral part of the platform. Indeed, the various aspects of trust are interrelated because, for example, refusing to participate in the dispute resolution process affects the user's reputation. Moreover, the user's ability to trade in the future will depend on his assessment and feedback. The level of participation on eBay is quite high, which means that buyers and sellers are actively using the rating system and often leave reviews. At the same time, statistics show that traders with a positive reputation are more likely to succeed in selling goods on the platform, which in turn allows eBay to exclude users with very low ratings from the market. This is a kind of natural selection. But again, it is important to take into account that the use of any digital platforms occurs at the initiative of the user himself. On any platform, there is necessarily a Terms of Use section, and if a new user accepts all rules, it means he gives voluntary consent to the opportunity to evaluate himself as a driver, passenger, seller or service provider. This is the key difference between objectification, which is often perceived in a negative way, and the term digitification, which does not carry a negative connotation.

Summing up the eBay rating system, it can be concluded that it gives a good visual representation of the seller's achievements and his selling experience. Those traders with better stars are usually more reliable. The rating increases the number of 
verified sellers and reduces the number of unreliable sellers on the platform, thus providing a quality product. Moreover, the rating system creates healthy competition, which also affects the quality and, of course, the price of the product.

\section{Services, rating and reviews. Yelp as one more digital platform with rating system}

The 21 st century is a time of new technologies and the Internet, and also a time when advertising and companies are less trusted than ever before. But at the same time, people trust ratings and reviews more than ever. Some studies show that about 85 percent of consumers say they use ratings and reviews to shop. And among consumers aged 45, 30 percent of consumers use ratings and reviews every time they want to buy something.

Another reason for the popularity of ratings is the two types of reviews that consumers pay attention to. There are third-party reviews such as Yelp and TripAdvisor, as well as top-notch reviews that are posted on the product site itself. Both are very important. Google primarily shows the sites of the products themselves and their ratings. This means that the more reviews a website has, the better it will be ranked.

From here another interesting fact comes - more and more businesses are politely and unobtrusively asking their customers to leave a review. Thus, lots of businesses try to come up with promotions and tricks to get as many reviews as possible. For example they give customers money as a coupon, an additional item or the second good as a gift.

But that actually has the opposite effect. New research in the Journal of Marketing Science finds that when you actually somehow pay customers to leave a review, they leave fewer reviews, because it makes people feel uncomfortable. Therefore, 
in order to gain as many reviews as possible, it is better not to pay customers, but make it easy to leave a review.

Another note: some really interesting research from Northwestern University found that the people who leave first party reviews, the people who leave reviews on your own website, are not the same people who use Yelp, TripAdvisor or any other third party platforms. There is actually a totally separate group of customers. This is great news for business, because it gets two sets of customer insights that can help this business build become better.

All of the above leads to another very important conclusion: honest reviews are objective and incorruptible. This thought makes the idea of a rating system accurate, and therefore confirms the benefits of its implementation and use.

\section{People's Republic of China as an example of embedded digitalization}

The People's Republic of China or simply PRC is a good example of digitalization as a social experiment in our world. This country has been practicing the Social Credit System for a relatively long time and its pilot has a successful experience and the basis for the permanent Social Credit System in the near future. The Social Credit System (the SoCS) - is a national blacklist being developed by the government of the People's Republic of China under General Secretary of the Chinese Communist Party Xi Jinping's administration. ${ }^{22}$ The program initiated regional trials in 2009, before launching a national pilot with eight credit scoring firms in 2014. ${ }^{23}$ It was first introduced formally by then Chinese Premier, Wen Jiabao, on October 20, 2011, during one of the State Council Meetings. ${ }^{24}$ In 2018,

\footnotetext{
22 "China rates its own citizens - including online behaviour". www.volkskrant.nl. 25 April 2015. Retrieved 26 December 2015.

${ }^{23}$ Zhong, Yuhao (Summer 2019). "Rethinking the Social Credit System: A Long Road to Establishing Trust in Chinese Society". Symposium on Applications of Contextual Integrity: 28-29 - via Privaci.info. 24 "What is China's social credit system and why is it controversial?". South China Morning Post. 9 August 2020. Retrieved 8 December 2020.
} 
these efforts were centralized under the People's Bank of China with participation from the eight firms. By 2020 it was intended to standardize the assessment of citizens' and businesses' economic and social reputation, or 'Social Credit'. ${ }^{25}$ The Social Credit Initiative calls for the creation of a unified citizen registration system, where both individuals and legal entities and their activities will be monitored. Initially, a digital system of rewards and punishments was meant, but recent studies have shown that the digital system was implemented only in a few regions as a pilot version, while the rest of the regions simply operate black and white lists that can be compared with a binary rating system.

Returning to the origins of the formation of CoSC it's worth saying that The World Bank acted as the ideologist of the credit rating implementation, motivating such a system with more flexible opportunities for national governments to dispose of available financial instruments. And already in the 21 st century, on the basis of the "bank rating", a whole "social rating system" was formed, the main ideologist of which was the PRC. In the Chinese interpretation, this system is formed from three main areas of human activity throughout his life:

1. Relations with the state

2. Behavior in society

3. Life in the digital space

The SoCS is often envisioned as a fully digitised and data-driven system. Indeed, digitalisation and informatisation are key items on China's political agenda, with the explicit goal to "use big data to modernise national governance", a theme set to feature prominently in the coming Five-Year Plan. However, it should be clear that

\footnotetext{
${ }^{25}$ Hatton, Celia (26 October 2015). "China 'social credit': Beijing sets up huge system". BBC News. Retrieved 23 December 2015.
} 
such digitalization does not carry a negative intent, but on the contrary, it is created in order to make the life of citizens safe, accessible and equal for everyone. ${ }^{26}$ As it is said in Planning Outline for the Construction of a Social Credit System (2014-2020) the Social Credit System is an important basis for comprehensively implementing the scientific development view and building a harmonious Socialist society, it is an important method to perfect the Socialist market economy system, accelerating and innovating social governance, and it has an important significance for strengthening the sincerity consciousness of the members of society, forging a desirable credit environment, raising the overall competitiveness of the country and stimulating the development of society and the progress of civilization. The main goals are:

1. Creating of sincerity culture and establishing mechanisms to encourage sincerity and punish insincerity

2. Building a mechanism to increase sincerity in government affairs, commercial sincerity, social sincerity and the authority of the judiciary, as well as significantly increase the level of satisfaction of the market and society, by comprehensively strengthening the sense of sincerity in the whole society

It is done so that any intruder (whether it is a citizen who doesn't repay the loan or runs the red light) would be sincerely ashamed to commit such an act next time, but it is necessary everyone knows about this violation.

The main idea is - it is not ashamed to steal something. It's a shame if everyone knows about it. This is the Chinese mentality and the Social Credit System is based on it.

\footnotetext{
${ }^{26}$ Yu, Qian 余茜 (2020). “十四五”治理有“数”开新篇 (In the "Fourteenth Five-Year Plan", governance will draw on "data" to open a new chapter)." October 28.
} 
The life of Chinese citizens before and after the Social Credit System is contrasted. There is no doubt that it has both advantages and disadvantages, but from a global point of view, the lives of citizens and many aspects of society have improved with the SoCS implementation. For clarity and accuracy, it is better to turn to social research. For example, as it is said in the research of Mercator Institute of China Studies - the Social Credit System is able to ensure stability. Indeed, China is a country with a fairly stable lifestyle. It is possible to observe here a constant growth in the production of goods and the development of the service market, due to which the standard of living is steadily increasing. China is the most powerful economy in the world. The education in this country is high quality, but at the same time affordable. And there are numerous legends about Chinese medicine.

The SoCS was even rapidly deployed to track and sanction violations of pandemic prevention measures, to stabilise prices and to later stimulate the safe and orderly return to the workplace. In various cities, citizens that attempted to evade quarantine, refused to have their temperature taken at checkpoints, or intentionally concealed their travel history to hard-hit areas were added to the blacklist. ${ }^{27}$ China as a country with its political regime and total submission is a big myth and delusion, and if you delve into this issue and thoroughly study the material part, you can understand that life in China is not at all the TV series "Black Mirror", where in one of episodes, the main character from vainly tries to earn points from friends, acquaintances, strangers passing by and even the store staff. This rating is subjective and based on personal perception. It carries no value, but only complicates life.

\footnotetext{
${ }^{27}$ Katja Drinhausen and Vincent Brussee. CHINA'S SOCIAL CREDIT SYSTEM IN 2021. From fragmentation towards integration. March 3, 2021.MERICS CHINA MONITOR. p.12
} 
In reality, a good citizen of the People's Republic of China must comply with certain rules and duties.

Every citizen must:

1. Pay taxes on time and repay the loan

2. Pay utility bills and payments by court order

3. Pay credit card bills on time

4. Comply with the traffic rules

5. Comply with the birth rate

6. Be honest in education and engage in social activities

7. Be polite on social networks and do not violate the boundaries of other people

8. Post reliable information on social networks

9. Have a good online shopping history and good buying habits

As it is seen from this list, all actions are legal and legitimate and correspond to the rights and obligations that any citizen is obliged to observe and fulfill. Nothing on the list is coercive and, as a result, does not violate anyone's rights.

While in the United States, Europe, Russia or any other countries the mass rating system is only gaining momentum and is gradually being implemented into many spheres of society with the advent of technology, the PRC is practicing the Social Credit System approach throughout the country en masse, and, most importantly, successfully, making life for Chinese people better every day. Sincerity culture breeds mutual respect and objectivity in their country, and therefore sets a good example for those who consider digitalization to be something negative. As well as digitalization in the PRC, the concept of digitification should be created in our modern world in order to make some things easier and more objective for everyone, as well as not to think stereotypically and primitively, guided by the rules of "good" and "bad". Digitification is not just the opposite of objectification. 
It is a deeper concept, created to refute the negative connotation of the word objectification and to bring to the world the idea that rating systems can have positive meaning and intention.

\section{Services for appearance assessment}

Today, anything can be evaluated, and this does not necessarily have a negative connotation. For example, there are several services on the Internet to assess attractiveness. Maybe some people will say that such services generate an objectification process in our society. But what is bad about the service for assessing one's appearance on a voluntary basis? The user independently comes to this service, provides his data, and accepts the cookie policy, as well as uploads a photo and accepts the terms of use. A person is interested in how attractive he is in the opinion of artificial intelligence. And if for the first user it is an opportunity to have fun, then for the second user it is an important aspect associated with, for example, professional activities.

For instance, a model will be seriously interested in assessing her appearance, because the better her makeup or hairstyle, the more chances she has to be cast and get a job. Or there is another example: a person is looking for a job and wants to make a good impression on the employer not only with his skills and abilities, but also with a presentable appearance. Why is it bad to choose the best photo using such a service? What kind of objectification is here?

One of these services is called photofeeler. This service helps users find out what impression their photos make. The opinion about the photo is based on the ratings of the customers of the service.

Before starting testing, the user must choose for what purposes he plans to use the photo: for dating, for business, or he will post the photo on social networks. Based on this, the frame will be evaluated according to different parameters. So, the 
opinion about the photo for the social network will be formed on the basis of how much other Photofeeler users consider the person in the photo to be cheerful, sincere and self-confident.

A chart is generated based on the scores of ten customers. It reflects the impression that photo creates.

\section{Credit Score in the United States}

Speaking about countries that have their own rating system, one cannot fail to mention the United States with its Credit Score.

A credit score is a number that provides a comparative estimate of an individual's creditworthiness based on an analysis of their credit report. ${ }^{28}$ Lenders, such as banks and credit card companies, use credit scores to evaluate the risk of lending money to consumers. Lenders contend that widespread use of credit scores has made credit more widely available and less expensive for many consumers. ${ }^{29}$ Under the Wall Street reform bill passed in 2010, a consumer is entitled to receive a free report of the specific credit score used if they are denied a loan, credit card or insurance due to their credit score. ${ }^{30}$

For the first time, scorecards appeared and began to be implemented by the United States in the 1940s. The points were put on the card by the credit expert based on his own experience, and about 10 characteristics were used for the assessment. In 1941, the first research paper on credit score was published by David Durand. In 1956, engineer Bill Fire and mathematician Earl Isaac developed the first scoring model and in the same year they created the Fair Isaac Corporation (FIC), which developed the FICO score. Consumers are often calculated according to the

\footnotetext{
${ }^{28}$ Kagan, Julia. "Credit Score". Investopedia. Retrieved 2021-05-24.

29 "Report to the Congress on credit scoring and its effects on the availability and affordability of credit".

30 "President Obama on Final Passage of Wall Street Reform: An End to Bailouts, a Beginning for Accountability". whitehouse.gov. July 15, 2010.
} 
consumer credit history database collected from three of the largest national bureaus: Experian, Equifax, and TransUnion.

The following aspects are taken into account: the quality of credit history, the presence and size of current debts, the duration of relations with creditors, the ratio of the number of applications submitted and issued loans, the types of loans issued. The borrower in accordance with this is assessed:

1. above 790 - "excellent"

2. 750-790 - average standard level

3. 740-750 - "very good"

4. 720-740 - "good"

5. 700-720 - "bad"

6. below 700 - "very bad"

For Americans, the median FICO score is 723.

Thus, the United States also has its own kind of rating system that rates borrowers in the field of lending. Such a system has been successfully operating in the USA for several decades and shows high quality of work. This once again confirms the effectiveness of rating systems.

\section{Conclusion}

In this article, both theoretical and practical materials are presented, so it is possible to see what a rating system and a rating scale are, what they are for and how they are used. Also in this work, assessment mechanisms for various online services and even the whole country are described, in order to show that the rating system does not carry any negative connotations. On the contrary it was created in order to provide people with the most reliable and accurate information, protect them and those who are around them, make their life comfortable and, finally, provide them with objective and useful information. 
In such conditions of the modern world, where everything is a subject to assessment and discussion, it is necessary to understand and be able to separate the "good" from the "bad" and the "negative" from the "positiv". So, if people have already learned how to rate goods from an online store using a binary, 5 or 10 point rating system, then there is still no idea for some of them how to evaluate human qualities. Moreover, a specific concept for such an assessment has not yet existed, and therefore this work provides clear arguments why the world community needs a new term which is called digitification and how it will affect people's lives. It is easy to rate something, but it is difficult to rate somebody. It is incorrect to rate a person in general, based on one thing only. It is also incorrect to rate a person based on someone's subjective perception only. For example, a woman cannot be regarded as an object of sexual desires just because the length of her skirt or dress does not correspond to generally accepted standards or personal perception. This is the process of objectification. People often do not know how to rate each other, and if they want to do it, they do not take into account many human qualities and characteristics, and therefore they materialize the object of assessment, reducing this process to something primitive and subjective.

The task of the new term digitification is to show that the assessment process is not always bad and does not always lead to objectification, because digitification is a voluntary assessment of the qualities of a subject based on objective criteria. From this, three key aspects can be distinguished:

\section{Voluntary}

2. Objective

\section{Assessment of qualities}

In the process of digitification, a person clearly understands that he is not evaluating another person, but his qualities and skills. Such an assessment process is voluntary, since a person himself decides to evaluate some of his attributes or 
allows another person or artificial intelligence to do it. And, finally, the assessment is based on objective criteria, previously created for a particular rating system.

For the new term digitification and its features, a full-fledged chapter is highlighted in this work, which gives a clear definition of the term and its characteristics. The introduction of a new term into everyday life can change the human perception of assessment for the better, open up new opportunities in the field of rating systems and services, and therefore improve people's lives, as it was defined earlier.

\section{References}

1. Arluke, Arnold (1988). "Sacrificial Symbolism in Animal Experimentation: Object or Pet?". Anthrozoös: A Multidisciplinary Journal of the Interactions of People and Animals. 2 (2): 98-117.

2. Nussbaum, Martha C. (1985). "Objectification". Philosophy \& Public Affairs. 24 (4): 249-291.

3. Nussbaum, Martha C. (1985). "Objectification". Philosophy \& Public Affairs. 24 (4): 257.

4. Langton, Rae Helen (2009). Sexual Solipsism: Philosophical Essays on Pornography and Objectification (1st ed.). Oxford: Oxford University Press. pp. 228-229.

5. Barry, Kathleen (1984). "Pornography: the ideoloy of cultural sadism". In Barry, Kathleen (ed.). Female sexual slavery. New York London: NYU Press. p. 247.

6. Jhally, Sut (director) (1997). Dreamworlds II: desire, sex, power in music (Documentary). USA: Media Education Foundation. 
7. Schama, Simon (1989). "The cultural construction of a citizen: II Casting roles: children of nature". In Schama, Simon (ed.). Citizens: a chronicle of the French Revolution. New York: Knopf Distributed by Random House.

8. Heldman, Caroline (August 2011). "Sexualizing Sarah Palin: the social and political context of the sexual objectification of female candidates". Sex Roles. 65 (3): 156-164.

9. Lyon, Harold C. (1977). Tenderness Is Strength (first ed.). New York: Harper \& Row.

10. "Sports, gym classes, team initiations and events". Sensations4women.com. 26 January 1998. Retrieved 1 August 2012.

11. Teunis, Niels (May 2007). "Sexual objectification and the construction of whiteness in the gay male community". Culture, Health \& Sexuality. 9 (3): $263-275$.

12. Teunis, Niels (2007). "Sexual objectification and the construction of whiteness in the gay male community". Culture, Health \& Sexuality. 9 (3): $263-275$.

13. Neimark, Jill (1 November 1994). "The beefcaking of America". Psychology Today. Sussex Publishers. Retrieved 1 August 2012.

14. Davidson, M. Meghan; Gervais, Sarah J.; Canivez, Gary L.; Cole, Brian P. (April 2013). "A psychometric examination of the Interpersonal Sexual Objectification Scale among college men". Journal of Counseling Psychology. 60 (2): 239-250.

15. Dawson, Leanne (2015). "Passing and policing: controlling compassion, bodies and boundaries in Boys Don't Cry and Unveiled/Fremde Haut". Studies in European Cinema. 12 (3): 205-228. 
16. Zimmerman, Amanda; Dahlberg, John (1 March 2008). "The Sexual Objectification of Women in Advertising: A Contemporary Cultural Perspective". Journal of Advertising Research. 48 (1): 71-79.

17. Paul F.M. Krabbe, in The Measurement of Health and Health Status, 2017

18. Chan, Ngai Keung. 2019. The Rating Game: The Discipline of Uber's User-Generated Ratings. Surveillance \& Society 17(1/2): 185.

19. Chan, Ngai Keung. 2019. The Rating Game: The Discipline of Uber's User-Generated Ratings. Surveillance \& Society 17(1/2): 185. 20. Uber's Community Guidelines 2015. p. 5.

21. Chris Dunne. eBay Stars: What They Mean and How to Get Them. Blog.edesk.com.

22. "China rates its own citizens - including online behaviour". www.volkskrant.nl. 25 April 2015. Retrieved 26 December 2015.

23. Zhong, Yuhao (Summer 2019). "Rethinking the Social Credit System: A Long Road to Establishing Trust in Chinese Society". Symposium on Applications of Contextual Integrity: 28-29 - via Privaci.info.

24. "What is China's social credit system and why is it controversial?". South China Morning Post. 9 August 2020. Retrieved 8 December 2020.

25. Hatton, Celia (26 October 2015). "China 'social credit': Beijing sets up huge system". BBC News. Retrieved 23 December 2015.

26. Yu, Qian 余茜 (2020). “十四五”治理有“数”开新篇 (In the "Fourteenth Five-Year Plan", governance will draw on "data" to open a new chapter)." October 28.

27. Katja Drinhausen and Vincent Brussee. CHINA'S SOCIAL CREDIT SYSTEM IN 2021. From fragmentation towards integration. March 3, 2021. MERICS CHINA MONITOR. P.12

28. Kagan, Julia. "Credit Score". Investopedia. Retrieved 2021-05-24. 
29. "Report to the Congress on credit scoring and its effects on the availability and affordability of credit".

30. "President Obama on Final Passage of Wall Street Reform: An End to Bailouts, a Beginning for Accountability". whitehouse.gov. July 15, 2010. 